\title{
Field Excitations and Primordial Observables
}

\author{
Dustin Lorshbough* \\ UT Austin \\ E-mail: Lorsh@utexas.edu
}

\begin{abstract}
Cosmological perturbation theory allows us to use our understanding of quantum field theory in curved spacetime to explain cosmological observations. We discuss computing primordial observables from knowledge of the underlying quantum theory and how the computation may be modified in the presence of different types of field excitation mechanisms. Modified scalar and tensor fluctuation propagators are discussed in the context of excited initial states. Modified matter propagators are discussed in the context of inflationary spectator fields. We emphasize that a better understanding of the mechanisms which may excite primordial field fluctuations will help us to understand how robust the predictions of inflationary cosmology are.
\end{abstract}

Frank N. Bash Symposium 2015

18-20 October

The University of Texas at Austin, USA

${ }^{*}$ Speaker. 
The anisotropies in the cosmic microwave background are believed to be the result of primordial inflationary fluctuations which are usually attributed to a slowly rolling scalar field [1]. During inflation, fluctuations in the metric and matter fields must be formulated in a way which breaks the degeneracy between the two. This results in gauge invariant scalar, vector and tensor perturbations. The vector perturbations redshift with the expansion of the universe and are therefore usually negligible, the scalar fluctuations are related to the temperature fluctuations observed in the cosmic microwave background and the tensor fluctuations describe gravitational waves [2].

Primordial observables are defined in terms of quantum correlation functions which may be computed using the in-in formalism [3]. At present, the interesting observable quantities are the scalar power spectrum (two-point function of scalar fluctuations), the tensor power spectrum (twopoint function of tensor fluctuations) and the scalar bispectrum (three-point function of scalar fluctuations) [4]. The tensor power spectrum and scalar bispectrum have not yet been measured. It is hoped that these and other correlation functions may be probed with future experiments [5, 6].

The computation of the primordial correlation functions is sensitive to the propagators of the scalar and tensor fluctuations, as well as the propagators of any other fields present during inflation which are coupled to gravity (called spectator fields if they do not help inflate). For the standard single field inflation predictions, the propagators of the scalar and tensor fluctuations are usually assumed to be the propagators corresponding to the lowest energy state in de Sitter space and the spectator fields which may be present during inflation are neglected altogether. However, it is interesting to see how robust the predictions of single field inflation are by considering more general propagators for the scalar, tensor and spectator field fluctuations.

The physical motivation for considering excited scalar and tensor fluctuations is that we are ignorant about the physics that proceeded the inflationary era. Several well-known pre-inflationary mechanisms such as tunneling transitions and fast roll to slow roll transitions result in fluctuations which are excited when inflation begins. For initial states which are a Bogoliubov transformation of the lowest energy vacuum state, it has been shown that the change in predicted quantities for scalar fluctuations is small since the amplitude of excitation is bounded by the observed scale invariance of the scalar power spectrum $[7,8,9]$. The tensor spectrum has not yet been detected so a similar argument for the bound on the tensor excitation amplitude cannot be made.

Spectator fields present during inflation are usually assumed to be in their lowest energy state, resulting in their contribution to primordial observables to be negligible compared to the leading order correlation function contribution. However, the propagators for the spectator fields may be enhanced due to physics before or during inflation allowing them to generate signatures which are potentially observable. One example of spectator field propagator enhancement is a scalar field with a time dependent mass which momentarily vanishes, this has been shown not to generically have interesting observable effects $[10,11,12,13,14,15]$. Another example is vector fields in the presence of a slowly rolling pseudoscalar field. The vector field fluctuations experience a temporary instability which exponentially enhances their amplitude, allowing them to have a non-negligible contribution to the primordial tensor spectrum and still be consistent with present observational data $[12,14,16,17,19,18]$.

We have outlined some of the possible modifications to the usual inflationary model which may affect primordial observables. Understanding more mechanisms for enhancing field fluctuations and their associated constraints will help us to understand the robustness of inflationary predictions. 


\section{References}

[1] D. Baumann and L. McAllister, Inflation and String Theory, arXiv:1404.2601 [hep-th].

[2] K. A. Malik and D. Wands, Cosmological perturbations, Phys. Rept. 475, 1 (2009) [arXiv:0809.4944 [astro-ph]].

[3] S. Weinberg, Quantum contributions to cosmological correlations, Phys. Rev. D 72, 043514 (2005) [hep-th/0506236].

[4] P. A. R. Ade et al. [Planck Collaboration], Planck 2015 results. XX. Constraints on inflation, arXiv:1502.02114 [astro-ph.CO].

[5] P. Creminelli, D. L. López Nacir, M. Simonović, G. Trevisan and M. Zaldarriaga, Detecting Primordial B-Modes after Planck, JCAP 1511, no. 11, 031 (2015) [arXiv:1502.01983 [astro-ph.CO]].

[6] S. Renaux-Petel, Primordial non-Gaussianities after Planck 2015: an introductory review, Comptes Rendus Physique 16, 969 (2015) [arXiv:1508.06740 [astro-ph.CO]].

[7] A. Aravind, D. Lorshbough and S. Paban, Non-Gaussianity from Excited Initial Inflationary States, JHEP 1307, 076 (2013) [arXiv:1303.1440 [hep-th]].

[8] R. Flauger, D. Green and R. A. Porto, On squeezed limits in single-field inflation. Part I, JCAP 1308, 032 (2013) [arXiv:1303.1430 [hep-th]].

[9] A. Aravind, D. Lorshbough and S. Paban, Bogoliubov Excited States and the Lyth Bound, JCAP 1408, 058 (2014) [arXiv:1403.6216 [astro-ph.CO]].

[10] J. L. Cook and L. Sorbo, Particle production during inflation and gravitational waves detectable by ground-based interferometers, Phys. Rev. D 85, 023534 (2012) [Phys. Rev. D 86, 069901 (2012)] [arXiv:1109.0022 [astro-ph.CO]].

[11] L. Senatore, E. Silverstein and M. Zaldarriaga, New Sources of Gravitational Waves during Inflation, JCAP 1408, 016 (2014) [arXiv:1109.0542 [hep-th]].

[12] N. Barnaby, J. Moxon, R. Namba, M. Peloso, G. Shiu and P. Zhou, Gravity waves and non-Gaussian features from particle production in a sector gravitationally coupled to the inflaton, Phys. Rev. D 86, 103508 (2012) [arXiv:1206.6117 [astro-ph.CO]].

[13] D. Carney, W. Fischler, E. D. Kovetz, D. Lorshbough and S. Paban, Rapid field excursions and the inflationary tensor spectrum, JHEP 1211, 042 (2012) [arXiv:1209.3848 [hep-th]].

[14] O. Özsoy, K. Sinha and S. Watson, How Well Can We Really Determine the Scale of Inflation?, Phys. Rev. D 91, no. 10, 103509 (2015) [arXiv:1410.0016 [hep-th]].

[15] M. Mirbabayi, L. Senatore, E. Silverstein and M. Zaldarriaga, Gravitational Waves and the Scale of Inflation, Phys. Rev. D 91, 063518 (2015) [arXiv:1412.0665 [hep-th]].

[16] S. Mukohyama, R. Namba, M. Peloso and G. Shiu, Blue Tensor Spectrum from Particle Production during Inflation, JCAP 1408, 036 (2014) [arXiv:1405.0346 [astro-ph.CO]].

[17] R. Z. Ferreira and M. S. Sloth, Universal Constraints on Axions from Inflation, JHEP 1412, 139 (2014) [arXiv:1409.5799 [hep-ph]].

[18] S. Eccles, W. Fischler, D. Lorshbough and B. A. Stephens, Vector field instability and the primordial tensor spectrum, arXiv:1505.04686 [astro-ph.CO].

[19] R. Namba, M. Peloso, M. Shiraishi, L. Sorbo and C. Unal, Scale-dependent gravitational waves from a rolling axion, arXiv:1509.07521 [astro-ph.CO]. 